\title{
Ganoderma lucidum Ameliorates Neurobehavioral Changes and Oxidative Stress Induced by Ethanol Binge Drinking
}

\author{
Chirlene Pinheiro Nascimento, ${ }^{1}$ Diandra Araújo Luz, ${ }^{1}$ Carla Cristiane Soares da Silva, ${ }^{1}$ \\ Cláudia Marques Rosa Malcher, ${ }^{1}$ Luanna Melo Pereira Fernandes, ${ }^{1}$ Herta Stutz Dalla Santa, ${ }^{2}$ \\ Antônio Rafael Quadros Gomes, ${ }^{1}$ Marta Chagas Monteiro $\mathbb{D D}^{1}{ }^{1}$ \\ Carolina Heitmann Mares Azevedo Ribeiro, ${ }^{3}$ Enéas Andrade Fontes-Júnior (D, 1 \\ and Cristiane Socorro Ferraz Maia $\mathbb{D}^{1}$ \\ ${ }^{1}$ Faculty of Pharmacy, Institute of Health Sciences, Federal University of Pará, Belém, 66075-900 Pará, Brazil \\ ${ }^{2}$ State University of Central-West, Department of Food Engineering (DEALI), Cedeteg Campus, Vila Carli, R. Simeão Varela de Sá, \\ Guarapuava, 85040-080 Paraná, Brazil \\ ${ }^{3}$ Laboratory of Immunology, Pharmacy Faculty, Institute of Health Science, Federal University of Pará, Belém, PA, Brazil
}

Correspondence should be addressed to Cristiane Socorro Ferraz Maia; crismaia@ufpa.br

Received 13 February 2020; Revised 8 May 2020; Accepted 26 May 2020; Published 31 July 2020

Guest Editor: Anderson J. Teodoro

Copyright (c) 2020 Chirlene Pinheiro Nascimento et al. This is an open access article distributed under the Creative Commons Attribution License, which permits unrestricted use, distribution, and reproduction in any medium, provided the original work is properly cited.

\begin{abstract}
Ganoderma lucidum, mushroom used for centuries by Asian peoples as food supplement, has been shown interesting biological activities, including over the Central Nervous System. Besides, these mushroom bioactive compounds present antioxidant and anti-inflammatory activities. On the side, binge drinking paradigm consists of ethanol exposure that reflects the usual consumption of adolescents, which elicits deleterious effects, determined by high ethanol consumption, in a short period. In this study, we investigated whether the Aqueous Extract of G. lucidum (AEGl) reduces the behavioral disorders induced by alcohol. Male $(n=30)$ and female Wistar rats $(n=40)$, seventy-two days old, were used for behavioral/biochemical and oral toxicity test, respectively. Animals were exposed to 5 binges (beginning at 35 days old) of ethanol $(3 \mathrm{~g} / \mathrm{kg} / \mathrm{day})$ or distilled water. Twenty-four hours after the last binge administration, animals received AEGl $(100 \mathrm{mg} / \mathrm{kg} / \mathrm{day})$ or distilled water for three consecutive days. After treatment protocol, open field, elevated plus maze, forced swim, and step-down inhibitory avoidance tests were performed. Oxidative stress parameters were measured to evaluate the REDOX balance. Our results demonstrated that AEGl elicited the recovery of spontaneous horizontal exploration capacity, anxiogenic- and depressive-profile, as well as short-term memory damage induced by binge-ethanol exposure. The behavioral effects of the extract were associated to the reequilibrium of the animals' REDOX balance. Thus, AEGl, a medicinal mushroom, ameliorates behavioral alteration on a model of motor, cognitive and psychiatric-like disorders induced by binge drinking paradigm and emerges as a useful tool as a food supplement in the management of disorders of alcoholic origin.
\end{abstract}

\section{Introduction}

Ethanol is a psychoactive drug able to alter several body systems, including the central nervous system (CNS). The exact molecular mechanisms associated to these injuries are still poorly understood, even though studies indicate the involvement of the certain mechanisms, such as the neuroinflammation and oxidative stress, on the neuropathological processes
[1-4]. The injury mechanisms seem to be related to the pattern of consumption $[5,6]$. Binge drinking is a pattern that consists of acute intoxication, i.e., 5 or more doses for men and 4 or more doses for women, during a short period of 2 hours, approximately, reaching a minimum blood alcohol concentration (BAC) of $80 \mathrm{mg} / \mathrm{dL}$ [6-8]. Previous researches carried out by our group have demonstrated that binge drinking pattern during adolescence modifies behavioral 
profiles, as spontaneous locomotion and motor coordination, as well as displays psychiatry-like and cognitive disorders, as anxiogenic and depressive phenotype, and memory impairment, associated to reduction-oxidation reactions (REDOX) imbalance in rodents $[9,10]$.

The knowledge of the probable mechanisms that underlie the ethanol tissue damage provides critically the search for alternatives managements to reduce these injuries, since there is still no adequate therapeutic management [11]. A promising alternative would be the use of therapeutic supplementation that consists of the use of bioactive functional foods, since they offer lower health risks and intoxication [12-14].

Actually, the use of dietary supplementation has increased, focused on reducing the onset or even treat diseases such as cancer, cardiovascular diseases, and neurological disorders ([12]., $[14,15])$. Some studies support the use of functional products for neurochemical changes associated to cognitive improvement, mood, and aging [12, 15]. In this regard, the mushroom Ganoderma lucidum (W. Curt.:Fr.) P. Karst. (Ganodermataceae, Agaricomycetes) emerges as an important source of bioactive compounds that can be used in the diet as supplementation, aiming to improve health $[16,17]$. Known as the "elixir of life" or "mushroom of immortality," officially used as a dietary supplement, this mushroom presents popular indications for "improving memory," "retarding senility," among other purposes [18-21]. Bioactive compounds such as triterpenes, lectins, and polysaccharides have been described in their composition [21-24].

A recent study reported that $G$. lucidum is able to act on free radicals and suppress lipid peroxidation, normalizing the balance between antioxidant defenses and reactive oxygen species, thus reducing oxidative stress [19]. In fact, studies have confirmed therapeutic activities of G. lucidum in the CNS, such as antidepressant action, cognitive improvement, and hypnotic properties, but the mechanisms have not yet been elucidated $[22,24]$.

In this sense, we hypothesize that pharmacological and biochemical activities from bioactive compounds of natural products, as G. lucidum, may diminish behavioral alterations induced by alcohol disorders. Thus, this work aims at evaluating the behavioral responses and the oxidative balance of G. lucidum in adult rats submitted to the binge drinking paradigm.

\section{Material and Methods}

2.1. Animals. Male Wistar rats, thirty-five days old at the beginning of the experiment, were used for behavioral/oxidative stress analyses. Female Wistar rats, seventy-two days old, were used for oral toxicity test. The animals were maintained at collective cages ( 5 animals per cage) to avoid stress by isolation and kept in controlled conditions of temperature $\left(23 \pm 1^{\circ} \mathrm{C}\right)$, exhaustion, light/dark cycle (12 h; lights ON 7 AM), water, and food ad libitum. All procedures were approved by the Ethics Committee on Experimental Animals of the UFPA (BIO-CEPAE 09-15), in accordance with the criteria established by the NIH Guide for the Care and Use of Laboratory Animals.
2.2. Preparation of Aqueous Extract of Ganoderma lucidum (AEGl). The lyophilized G. lucidum mycelium, provided by BRASMICEL-SP was deposited on the Micoteca of the Laboratório de Bioprocessos de Cogumelos-DEALI (UNICENTRO, Guarapuava, Paraná, Brazil) and triturated, mixed with distilled water and homogenized in the vortex for five minutes. The resulting suspension was filtered and administered orally at a final volume of $0.1 \mathrm{ml}$ for each $100 \mathrm{~g}$ of animal weight, equivalent to $100 \mathrm{mg} / \mathrm{kg} /$ day [25].

2.3. Binge Drinking Paradigm and AEGl Treatment. All administrations were performed by oral gavage with orogastric cannula (Insight, Brazil). It was classified as binge the pattern of three followed administrations of ethanol per week, once per day [9]. Three groups were used: control, untreated, and treated individuals. The treated and untreated groups were exposed to 5 binges (beginning at 35 days old) of ethanol $(3 \mathrm{~g} / \mathrm{kg} / \mathrm{day} ; 20 \% \mathrm{w} / \mathrm{v})$, which consists the middle adolescence until the adult phase [10]. This binge-drinking paradigm reaches a BAC of approximately $250 \mathrm{mg} / \mathrm{dL}$ [10], which reflects the binge-drinking pattern (over $80 \mathrm{mg} / \mathrm{dL}$; [8]). Twenty-four hours after the last binge administration, animals received AEGl (treated) or distilled water (untreated) for three consecutive days (Figure 1).

2.4. Oral Toxicity Assay. The oral toxicity test was based on OECD Guideline 420, with adaptations. Female Wistar subjects were divided into four groups. The test groups ( $N=15 /$ group) received orally an acute dose of $2,000 \mathrm{mg} / \mathrm{kg}$ or $5,000 \mathrm{mg} / \mathrm{kg}$ of $\mathrm{G}$. lucidum, while the control group $(N=5$ animals/group) received distilled water in an equivalent volume. Animals were evaluated as predicted by the Behavioral Pharmacological Triage [26]. Animal mortality was observed after $30 \mathrm{~min}, 1 \mathrm{~h}, 2 \mathrm{~h}$, and $24 \mathrm{~h}$ of administration. These observations were maintained up to 14 days after the administration of the G. lucidum, once a day and always at the same time, for 30 minutes. Following behavioral toxicity evaluation, animals were sacrificed by cervical displacement followed by cardiac puncture for blood collection and hematological and oxidative biochemistry analysis on the first day (D1) and other groups on the fourteenth day (D14) after protocol administration.

2.5. Behavioral Evaluations. Previously to behavioral tests, animals were conducted for the behavioral experimental room for habituation for $2 \mathrm{~h}$ that consists of a low-intensity light (12lx) and sound-attenuated room. All experimental procedures were carried out between 9:00 AM and 4:00 PM.

2.5.1. Open Field Test (OF). To perform the test, a black acrylic arena (100 x $100 \mathrm{~cm}$ wide and $40 \mathrm{~cm}$ high) was used. Briefly, animals were placed individually on the center of the apparatus, and free movement was allowed for 5 minutes. The test was videotaped for further analysis by ANY-maze TM (Stoelting, USA). The motor parameters (i.e., total distance traveled and number of rearing) were measured [9].

2.5.2. Elevated Plus Maze (EPM) Test. Animals were submitted individually to an elevated wooden apparatus $50 \mathrm{~cm}$ from the floor, with two enclosed arms and two open arms 


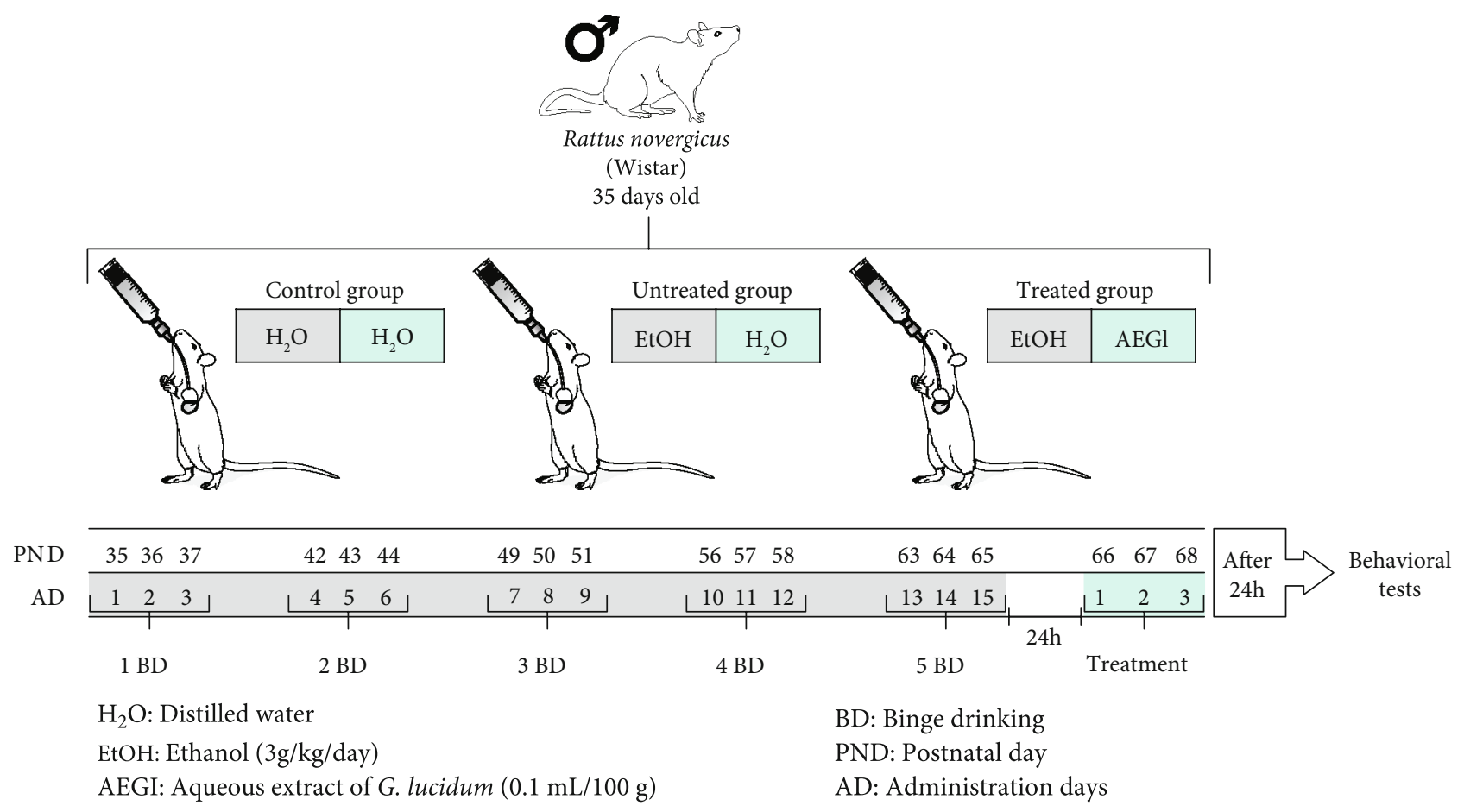

FIGURE 1: Experimental design of the study.

$(50 \times 10 \mathrm{~cm})$ opposite each other. In the closed arms, there are lateral walls of $40 \mathrm{~cm}$ of height. Each animal was placed on the center of the EPM facing one of the closed arms, being allowed to explore the equipment for 5 minutes. The percentage of time spent in the open arms (\%OAT), the percentage of open arms entries (\%OAE), and the number of entries in the closed arms (EAE) was counted. The \%OAE and $\% \mathrm{OAT}$ were calculated according to the formula [(open/ total $\times 100$ ]. An entry was counted whenever the animal placed four paws in an arm of the maze [9].

2.5.3. Forced Swimming (FS) Test. The apparatus of the forced swimming test (FS) consists of a $50 \mathrm{~cm}$ high and $30 \mathrm{~cm}$ diameter cylinder containing $40 \mathrm{~cm}$ of water column at a temperature of $23 \pm 1^{\circ} \mathrm{C}$. Animals were placed on the center of the equipment for 5 minutes. The initial 2 min consisted of the habituation phase. In the final three minutes, immobility time (brief movements to keep floating) was measured $[9,27]$.

2.5.4. Step-Down Inhibitory Avoidance (IA) Test. The test was carried out in an aluminum box $(50 \times 50 \times 35 \mathrm{~cm})$, containing one safe platform and a floor consisting of 15 parallel copper bars connected to an electric stimulator. Briefly, the animals were habituated for 180 seconds to the apparatus, allowing free exploration. After 24 hours, the animals were submitted to the training session and reexposed to the safe platform of the apparatus. Immediately after the animal step-down from the platform with the four legs on the grids, an electric shock of $0.4 \mathrm{~mA}$ for 1 second (aversive stimulus) was applied. Each animal was reexposed again $1.5 \mathrm{~h}$ after the training to verify short-term memory acquisition. Thus, the latency to step- down from the secure platform was recorded as the level of short-term memory retention [9].

\subsection{Oxidative Biochemistry Assays}

2.6.1. Determination of Plasma Malondialdehyde (MDA) Concentration. MDA dosage was based on the formation of the complex with thiobarbituric acid with MDA. The TBA was prepared according to enterprise guideline (Sigma Aldrich, Germany). Briefly, $1 \mathrm{~mL}$ of TBA solution (10 nM) plus $500 \mu \mathrm{L}$ of the plasma sample was mixed. The TBAMDA complex levels were detected by the spectrophotometer device (wavelength $535 \mathrm{~nm}$ ) [28].

2.6.2. Measurement of Trolox Equivalent Antioxidant Capacity (TEAC). TEAC evaluation consists of measuring the inhibition of the ABTS+• cation (2,2-Azinobis- [3-ethylbenzothiazoline-6-sulphonate radical, diammonium salt) in the sample. Briefly, $2970 \mu \mathrm{L}$ of working solution was added to the cuvette, followed by reading the absorbance spectrophotometer. This value corresponds to T0. Subsequently, $30 \mu \mathrm{L}$ of the homogenized plasma sample was added and the timer was activated to mark 5 minutes. After 5 minutes, the absorbance, corresponding to T5, was recorded [29]. The calculation of the results was obtained from the use of formulas based on the absorbance results of the A-F tubes, curve standard $\left(R^{2}=0.9964 ; y=0.01732 \mathrm{x}-0.1367\right)$ to obtain the calculation of the Trolox equivalent sample, in the following order: $\mathrm{TAAc}=(\mathrm{T} 0+\mathrm{T} 5 / \mathrm{T} 0)-$ White control TAA and TEAC $=($ TAAc -0.1367$) / 0.01732$.

The total antioxidant potential of serum determined as $\mu \mathrm{AC} / \mathrm{mL}$ TEAC and calculated by a calibration curve with different amounts of Trolox $[29,30]$. 
2.6.3. Measurement of Superoxide Dismutase (SOD) Activity. SOD dosage was based on the competition of SOD with XOD (xanthine oxidase enzyme) by the superoxide radical. A $100 \mu \mathrm{L}$ aliquot of each blood sample was mixed with $400 \mu \mathrm{L}$ of ice water to promote hemolysis. The volume of $3 \mathrm{~mL}$ was taken for reading in the cuvettes by a spectrophotometer $(550 \mathrm{~nm})$ in the time of 5 minutes. The absorbance values were recorded from time 0 (T0) and time 5 (T5). T0 was noted before the addition of the XOD reagent, then, the XOD was added and counted for 5 minutes to check the final reaction (T5). The final calculation considered: $\mathrm{SOD}=(\%$ inhibition $) \mathrm{x}$ (Dilution factor $) /(50 \%) \mathrm{x}(0.10)$, which $50 \%$ is the reduction rate of Cytochrome $\mathrm{C}$ by unit definition; and 0.10 the volume in milliliters of the sample used in each test [31]. Final results were expressed in units $/ \mathrm{mL}$.

2.6.4. Measurement of Total Glutathione (GSH). GSH analysis was based on the ability of the GSH present in the sample in reduce 5,5-dithiobis-2-nitrobenzoic acid (DTNB) to nitrobenzoic acid (TNB). The sample was read by the spectrophotometer at $412 \mathrm{~nm}$ of wavelength. After hemolysis generated by the mixture of $20 \mu \mathrm{L}$ of the total blood and $180 \mu \mathrm{L}$ of distilled water, $20 \mu \mathrm{l}$ of the hemolysate was added in $20 \mu \mathrm{l}$ of distilled water plus $4 \mu \mathrm{L}$ of PBS/EDTA and vortexed. An aliquot of $3 \mathrm{~mL}$ of the sample was placed in the spectrophotometer reading cuvette, and the absorbance at time 0 (T0) was measured. Then, $100 \mu \mathrm{L}$ of the DTNB was added and the time count was started for 3 minutes. The reading time of 1 minute (T1) and 3 minutes (T3) from the addition of the DTNB was recorded [32]. To calculate the GSH concentration in the sample, the equation of a standard curve previously performed $\left(y=154.38 \mathrm{x}-3.2983 ; R^{2}=0.9421\right)$ was used. The concentrations of GSH in the samples were expressed in $\mu \mathrm{g} / \mathrm{mL}$.

2.7. Statistical Analyses. Results are expressed as mean \pm S.E.M. of $n=6-10$ animals/group for behavioral analysis and $n=4-7$ /group for oxidative biochemical assays. Statistical comparison was performed by one-way ANOVA, with multiple post hoc comparisons of Tukey's test for behavioral analysis or Bonferroni test for biochemical assays, considering a significance level of 95\%. GraphPad Prism 7.0 software was employed to perform statistical analyzes and graphical building.

\section{Results}

3.1. AEGl Presents Low Toxicity. AEGl (1000 and $5000 \mathrm{mg} / \mathrm{kg}$ ) administered acutely by oral route did not promote deaths during the acute oral toxicity assay period. However, such high doses resulted in sedative effects (Table 1), changes in oxidative balance (Table 2), and neutropenia (Table 3).

3.2. AEGl Recovers the Ability of Vertical Exploration of the Animals Previously Intoxicated by Ethanol in Pattern Binge in the of Test. To evaluate the activity of spontaneous locomotion in rodents, we can benefit from the natural tendency of animals of exploring new environments [33]. Motor injuries are a common impairment that occurs after ethanol intoxication in a binge pattern $[9,10]$. For this evaluation,
TABLE 1: Behavioral screening after acute administration of Aqueous Extract of Ganoderma lucidum (AEG1) by gavage in adult female Wistar rats in the acute oral toxicity test. A = EAGl $2000 \mathrm{mg} / \mathrm{kg}$; B = EAGl $5000 \mathrm{mg} / \mathrm{kg}$; and C = control groups. $(+)$ Presence of the behavior signals; (0) Absence of behavior signals.

\begin{tabular}{|c|c|c|c|c|c|c|c|c|c|}
\hline \multirow{3}{*}{ Signal } & \multicolumn{9}{|c|}{ Time after administration } \\
\hline & \multicolumn{3}{|c|}{$30 \mathrm{~min}$} & \multicolumn{3}{|c|}{$1 \mathrm{~h}$} & \multicolumn{3}{|c|}{$2 \mathrm{~h}$} \\
\hline & A & B & $\mathrm{C}$ & A & B & $\mathrm{C}$ & A & B & $\mathrm{C}$ \\
\hline Lethargy & + & + & 0 & + & + & 0 & + & + & 0 \\
\hline Ptosis & + & + & 0 & + & + & 0 & + & + & 0 \\
\hline Agitation & + & + & 0 & + & 0 & 0 & + & 0 & 0 \\
\hline Tremors & 0 & 0 & 0 & 0 & 0 & 0 & + & 0 & 0 \\
\hline Increase of grooming & 0 & + & 0 & 0 & 0 & 0 & 0 & 0 & \\
\hline \multirow[t]{2}{*}{ Increase of rearing } & 0 & + & 0 & 0 & 0 & 0 & 0 & 0 & \\
\hline & \multicolumn{9}{|c|}{ Days after administration } \\
\hline \multirow[t]{2}{*}{ Signal } & \multicolumn{3}{|c|}{ Day 2} & \multicolumn{3}{|c|}{ Day 4} & \multicolumn{3}{|c|}{ Day 14} \\
\hline & A & B & $\mathrm{C}$ & A & B & $\mathrm{C}$ & A & B & C \\
\hline Lethargy & + & + & 0 & 0 & + & 0 & 0 & 0 & 0 \\
\hline Mortality & 0 & 0 & 0 & 0 & 0 & 0 & 0 & 0 & 0 \\
\hline
\end{tabular}

the OF test was performed. The AEGl $(0.1 \mathrm{~mL} / 100 \mathrm{~g})$ recovered the reduction of total distance traveled displayed by binge drinking administration in rats $(P=0.0224$, Figure 2(a)), in which the animals performed the horizontal ambulation similar to the control group. There was no significant difference between the groups in the evaluation of the rearing number (Figure 2(b)), which refers to vertical exploitation.

3.3. AEGl Improves Emotional Behaviors Resulted from the Consumption of Ethanol in a Binge Pattern. Ethanol is able of generating emotional disturbances $[9,10]$. For the evaluation of emotional behaviors, i.e., anxiogenic- and depressive-type, the elevated plus maze (EPM) and forced swimming (FS) tests were performed, respectively. The former is based on the preference of the animals to dark, closed, and small places. Nevertheless, the animals still maintain the exploratory behavior [34]. The latter is known as a model of "behavioral desperation," in which the depressive-like profile animals exhibit elevated immobility time during the task $[27,35]$.

In the EPM test, animals that were submitted to the ethanol paradigm reduced the open arms time (\%OAT; Figure 3(b)), which suggests the anxiogenic-like phenotype. Besides, the enclosed arms entries (EAE) parameter was reduced in the ethanol-exposed animals, which confirms the reduction on spontaneous motor performance (Figure 3(c)). Therefore, animals that received the G. lucidum extract increased the $\% \mathrm{OAT}$, reaching the levels related to the control group $(P=0.0067)$, as well improved motor ambulation that corroborates with the findings found in OF test.

In the FS test, the AEGl administration reduced the immobility time, displayed by successive binge drinking exposure in rats (Figure $3(\mathrm{~d}) ; P=0.0110$ ), which reflects 
TABLE 2: Analysis of the ratio between total antioxidant capacity (TEAC) and malondialdehyde (MDA) levels in animals treated with EAGl at doses of 2000 and $5000 \mathrm{mg} / \mathrm{kg}$ on days 1 and 14 .

\begin{tabular}{lcccc}
\hline \multirow{2}{*}{ Group } & & \multicolumn{2}{c}{ Analyses period } & Day 14 \\
\hline AEGl $(2000 \mathrm{mg} / \mathrm{kg})$ & TEAC/MDA & Day 1 & TEAC/MDA & $P$ value \\
AEGl $(5000 \mathrm{mg} / \mathrm{kg})$ & $0.47 \pm 0.08^{*}$ & $<0.0001$ & $0.41 \pm 0.09^{*}$ \\
Control & $0.44 \pm 0.08^{*}$ & $<0.0001$ & $0.40 \pm 0.04^{*}$ & $<0.0001$ \\
\hline
\end{tabular}

TABLE 3: Analysis of hematological parameters in oral toxicity in the groups treated with EAGl at doses of 2000 and $5000 \mathrm{mg} / \mathrm{kg}$ and in the control group for 1 and 14 days. A = treated with EAGl $2000 \mathrm{mg} / \mathrm{kg}$; B = treated with EAGl $5000 \mathrm{mg} / \mathrm{kg}$; and C = control groups. Results expressed by mean \pm SEM of 5-8 animals per group (one-way ANOVA, Tukey's test). ${ }^{*} P<0.0001$ when compared to the control group.

\begin{tabular}{|c|c|c|c|c|c|c|}
\hline \multirow{3}{*}{ Cells (\%) } & \multicolumn{6}{|c|}{ Analyses period } \\
\hline & & Day 1 & & & Day 14 & \\
\hline & A & B & $\mathrm{C}$ & $\mathrm{A}$ & B & $\mathrm{C}$ \\
\hline Lymphocytes & $87.25 \pm 7.63^{*}$ & $79.75 \pm 4.75^{*}$ & $49.2 \pm 3.44$ & $84.7 \pm 2.9^{*}$ & $79 \pm 8.7^{*}$ & $52.1 \pm 8.4$ \\
\hline Neutrophils & $11.25 \pm 7.38^{*}$ & $18 \pm 4.5^{*}$ & $44.6 \pm 3.92$ & $14.7 \pm 3.1^{*}$ & $15.7 \pm 6.2^{*}$ & $41.8 \pm 7.9$ \\
\hline Monocytes & $1.5 \pm 0.5$ & $2.25 \pm 0.88$ & $3.2 \pm 1.04$ & $0.7 \pm 0.4$ & $3 \pm 1.3$ & $3.5 \pm 0.7$ \\
\hline
\end{tabular}

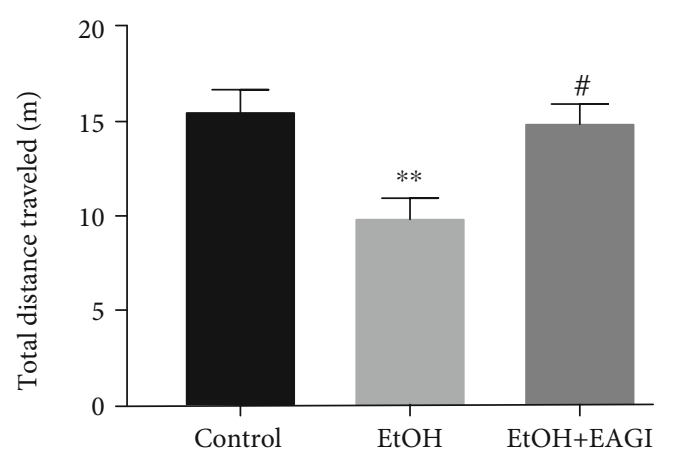

(a)

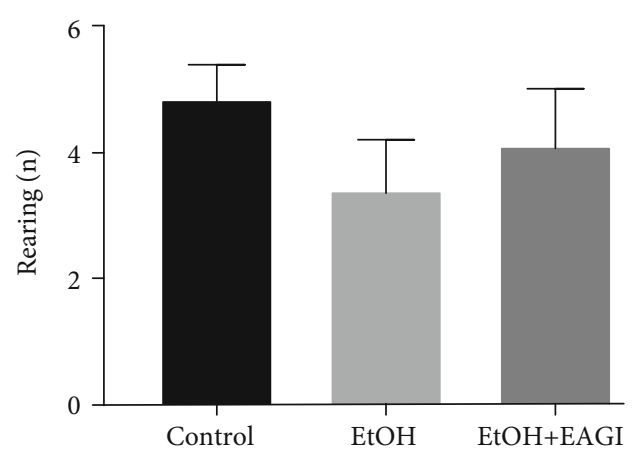

(b)

Figure 2: Effects of Aqueous Extract of Ganoderma lucidum (AEGl) on the spontaneous locomotion after the intoxication by ethanol in a binge pattern on adults' male rats. The analysis of the total distance traveled allowed to evaluate the horizontal exploitation of the animals (a), and the number of rearing as vertical exploitation (b), both related to motor activity of the animals. ${ }^{* *} P<0.01$ (compared to the control group); ${ }^{\#} P<0.05$ (compared to EtOH group). Data were analyzed by One-way ANOVA (mean \pm SEM; $n=7-10$ animals per group).

antidepressant activity. After the mushroom extract intake, ethanol-treated animals reached immobility time values related to control animals.

\subsection{AEGl Improves Short-Term Memory Damages Induced by} Ethanol Binge Drinking Exposure. Studies have revealed that binge drinking leads to short-term memory impairment [9]. To evaluate the short-term memory, we applied the inhibitory avoidance test (IA) that consists of obtaining the behavioral response from an aversive stimulus (i.e., footshock) [36, 37]. One hour and a half after the training session that was applied to the aversive stimulus, animals were submitted to the short-term memory test. Animals that were exposed to the binge drinking protocol reduced the latency to descend from the secure platform. However, individuals that received AEGl recovered the ethanol-induced mnemonic damage, increasing the latency to step-down from the secure platform of the apparatus $(P<0.0001$; Figure 4$)$.

3.5. G. Lucidum Extract Promotes the Equilibrium of the REDOX Balance. REDOX balance is mediated by the equilibrium between the generation of reactive oxygen and/or nitrogen species and the amount of antioxidant defenses. The disturbance on such factors induces to severe damage in the organism [38]. In general, the AEGl treatment reestablished the REDOX balance, which promoted a counterbalance between free radicals and antioxidant defenses in ethanoltreated subjects.

Figures 5(a)-5(d) show that G. lucidum extract reduced lipid peroxidation, as well as induced the equilibrium of the antioxidant defenses, such as GSH and TEAC, that returned to the oxidative parameters related to the basal levels 


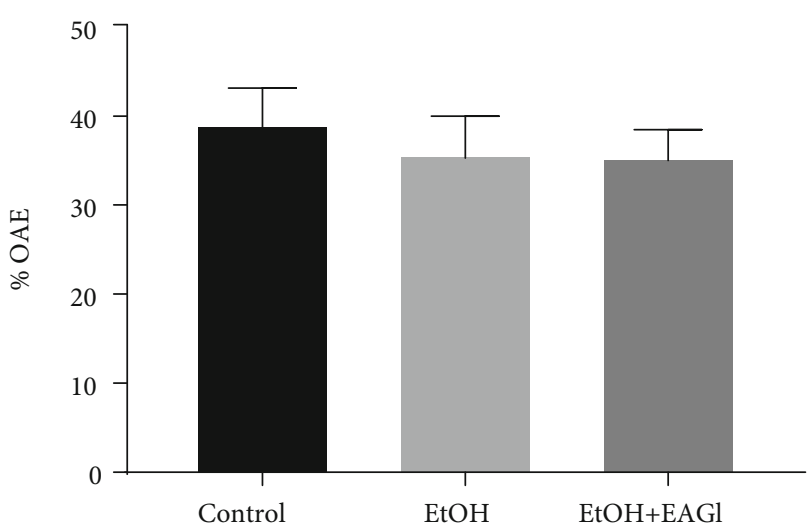

(a)

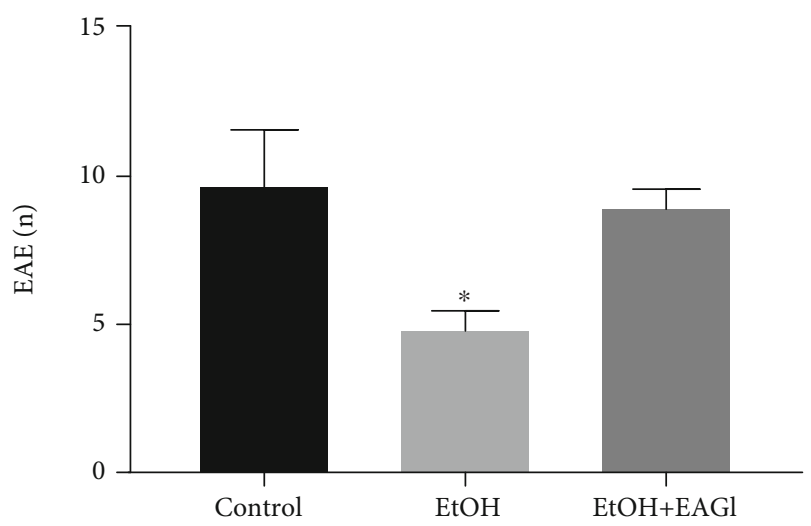

(c)

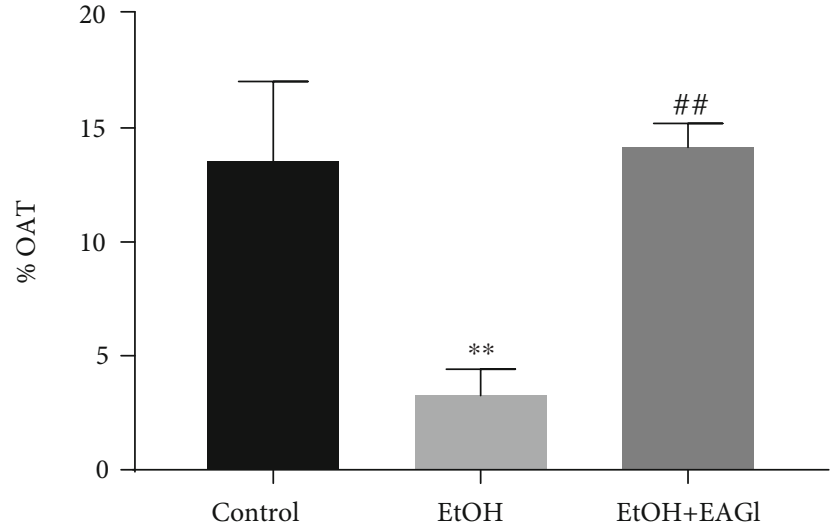

(b)

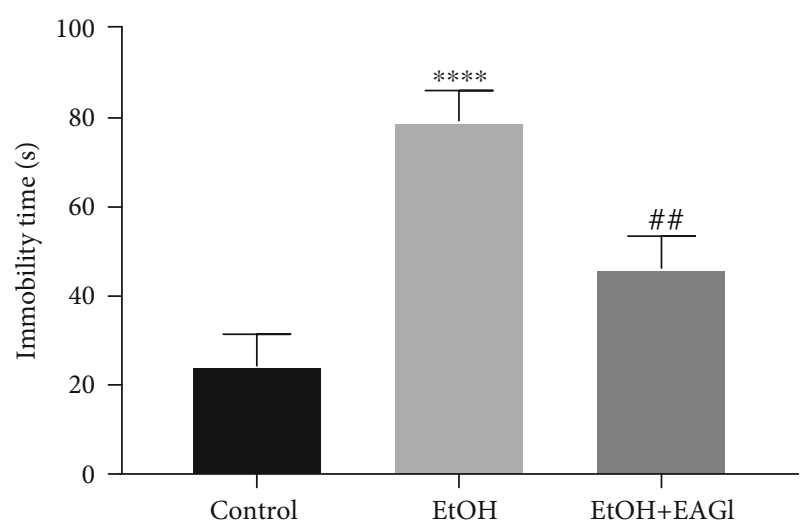

(d)

FIgURe 3: Effects of Aqueous Extract of Ganoderma lucidum on emotional alterations provoked by intoxication with ethanol (EtOH) in a binge pattern. The parameters percentage of entries in the open arms (a), percentage of time spent in the open arms (b), and the number of entries in the closed arms (c) allowed the assessment of the anxious behaviors and locomotor activity on the elevated plus-maze paradigm. Immobility time (d) parameters were admitted as an index of depressive-like behavior on forced swim test. ${ }^{*} P<0.05$ (compared to control group); ${ }^{* *} P<0.01$ (compared to control group); ${ }^{* * * *} P<0.0001$ (compared to control group); ${ }^{\# \#} P<0.01$ (compared to $\mathrm{EtOH}$ group). Data were analyzed by One-way ANOVA (mean \pm SEM; $n=6-9$ animals per group).

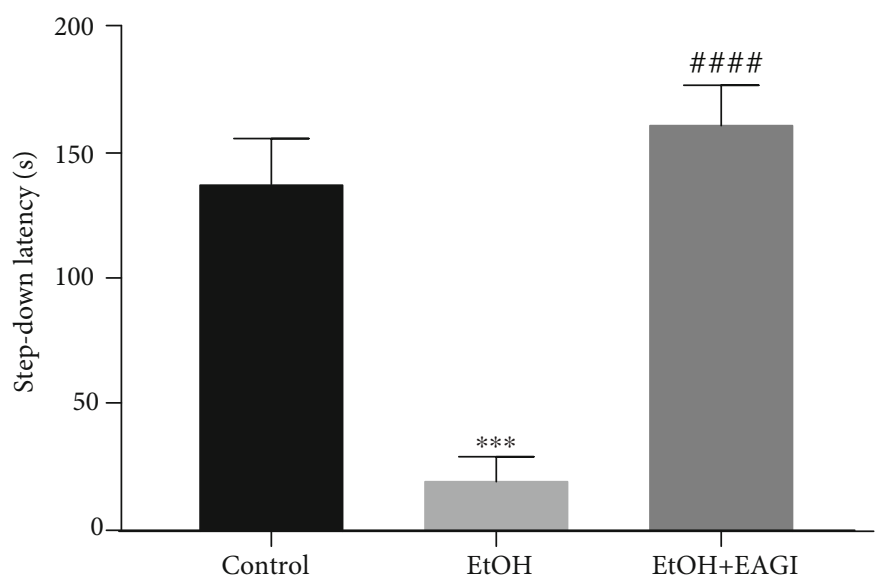

Figure 4: Effects of Aqueous Extract of Ganoderma lucidum (AEGl) on the short memory after the intoxication by ethanol (EtOH) in a binge pattern on adults' male rats. The step-down latency allowed to evaluate the short-term memory (1.5 h). ${ }^{* * *} P<0.001$ (compared to control group); ${ }^{\# \# \#} P<0.0001$ (compared to EtOH group). Data were analyzed by One-way ANOVA (mean \pm SEM; $n=6-7$ animals per group). 


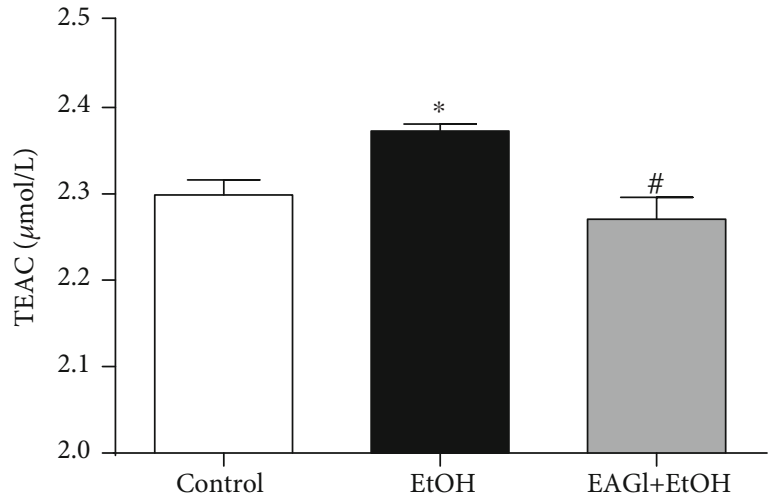

(a)

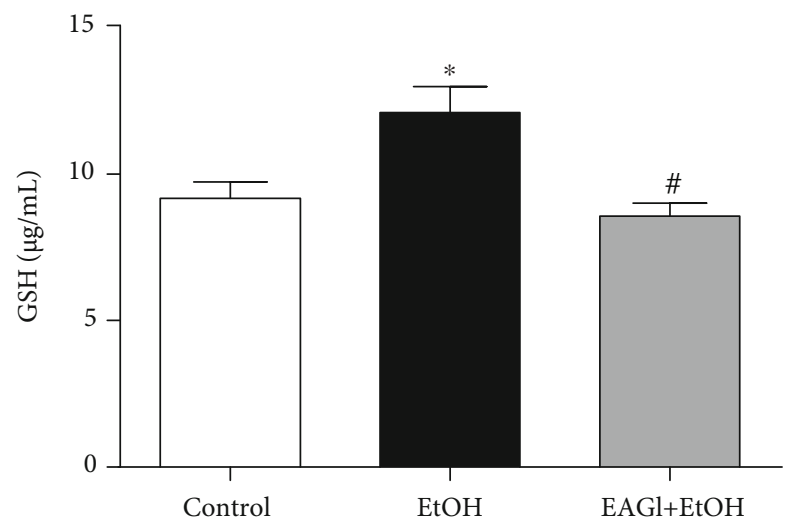

(c)

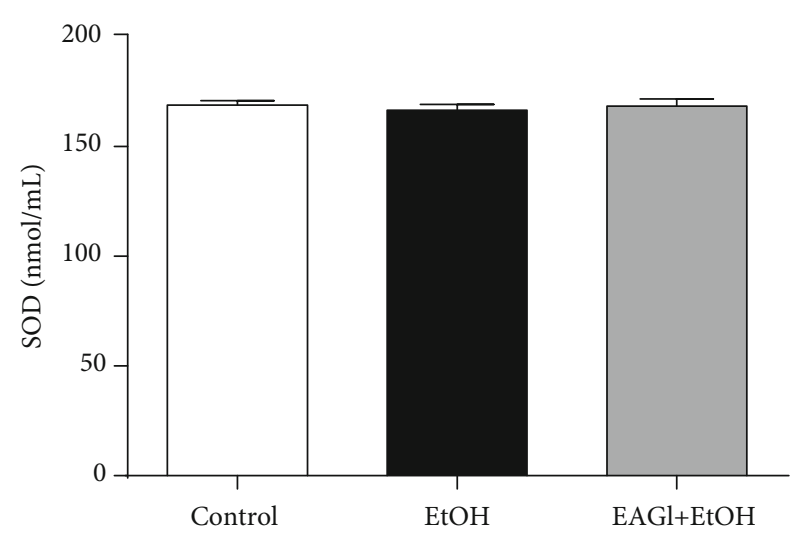

(b)

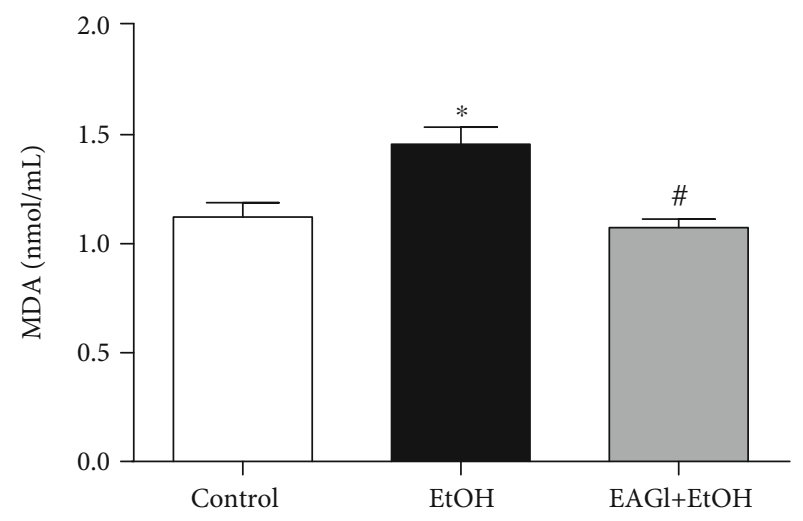

(d)

FIGURE 5: Effects Aqueous Extract of Ganoderma lucidum after intoxication by ethanol (EtOH) in a binge drinking pattern on the REDOX system. Total antioxidant capacity (TEAC; a); Superoxide dismutase (SOD) enzymatic activity (b); glutathione (c); and lipid peroxidation (MDA; d) were evaluated. ${ }^{*} P<0.05$ (compared to control group); ${ }^{\#} P<0.05$ (compared to EtOH group). Data were analyzed by One-way ANOVA (mean \pm SEM; $n=5$ animals per group).

expressed by the control group. In recent years, our group has shown that exposure to ethanol leads to an overproduction of reactive species and levels of antioxidant factors, in an attempt to compensate for the oxidative stress that causes damage to macromolecules, such as increased lipid peroxidation in the body.

\section{Discussion}

This study provides the first evidence that AEGl, a functional food, ameliorates behavioral alteration on a model of psychiatric-like disorders induced by binge drinking paradigm. Our results demonstrated the recovery of the spontaneous horizontal exploration capacity of the animals in the OF test, as well as extinguished the anxiogenic- and depressive-profile induced by ethanol exposure. On the cognitive field, the administration of AEGl reversed the ethanolinduced short-term memory damage. The behavioral effects of the extract were associated to the reequilibrium of the animals' REDOX balance.

Firstly, we submitted animals to the toxicological test. OECD guideline 423 was applied to verify the acute oral toxicity of AEGl [39]. Our results revealed that the Ganoderma extract did not show any lethality in the doses of 2,000 and $5,000 \mathrm{mg} / \mathrm{kg}$. According to OECD xenobiotics classification based on five levels of toxicity parameters scale [40], the AEGl toxicological findings establish the extract on the category 5 , which reflects the low toxicity xenobiotic, since the median lethal dose $\left(\mathrm{LD}_{50}\right)$ is over $5,000 \mathrm{mg} / \mathrm{kg}$. The characterization of these safety standards endorsed their application in pharmacological tests and the security for consumption.

Studies on toxicity, ability to cause harmful effects on living organisms, and adverse effects of G. lucidum are uncommon [41]. Acute oral toxicity is the method used to assess the toxicity produced by a substance when administered within 24 hours, followed by a 14-day observation, used to check and classify the substances as to their lethality, establishing safety parameters for human health, evaluating in animals the neurobehavioral alterations $[26,42]$, as well as hematological [43] and biochemical parameters [44].

We adopted, for this evaluation, the fixed-dose procedure suggested by OECD 423 [39]. We highlight that excessive doses of the AEGl (over $2,000 \mathrm{mg} / \mathrm{kg}$ ) may promote lethargy, oxidative unbalance, and hematological alterations, which emphasizes the care of the dose and time use of the extract. 
After that, we employed our ethanol-induced neurobehavioral impairment protocol. Recently, we showed that ethanol reduced the spontaneous locomotion of the individuals submitted to the binge-drinking protocol during adolescence $[9,10]$. Our results demonstrated that such an ethanol paradigm during the adult phase also induced the dulling of the exploitation. Regards emotionality, we also found that this same pattern display anxiogenic and depressant phenotype in adult rats. Our previous data on adolescent individuals have reported such psychiatric-like conditions $[9,10]$, however, this is the first time that we demonstrated that sequential binge drinking pattern ( 5 cycles of 3 on- 4 off) during adolescence until early-adult life induces anxiogenic- and depressant-like behavior in rodents.

In addition to emotionality, ethanol intake also negatively affects the mnemonic process. In this sense, our data demonstrated the impairment of short-term memory through an aversive stimulus paradigm in adult animals that have been already proposed by our group during adolescence period [9].

Actually, acute exposure to ethanol modifies the neurotransmission patterns, which occurs the overstimulation of GABAergic pathways, due to high $\mathrm{GABA}_{\mathrm{A}}$ receptor stimulation, as well as reduction of the glutamatergic pathway activity, related to the block of the NMDA glutamate receptors [45]. Besides, other neurotransmission pathways are also affected, i.e., serotonergic and dopaminergic, which result in alterations of behavioral responses $[46,47]$.

However, ethanol long-term exposure and withdrawal display compensatory physiological mechanisms to balance neurotransmissions, through negative regulation of $\mathrm{GABA}_{\mathrm{A}}$ receptors, increased expression of NMDA receptors and loss of glutamate reuptake glial function, which results in overstimulation of CNS, known as excitotoxicity [46, 48-51].

Excitotoxicity leads to neuroglial activation and consequent neuronal death in the most varied CNS regions, culminating in the generation of reactive oxygen species (ROS), which in turn produces unbalance in the REDOX system and oxidative stress [52]. On the other hand, oxidative stress results in late inflammatory response due to oxidative damage, further increasing the production of ROS, which, in turn, accentuates the inflammatory process, that leads to chronic inflammatory and oxidative stress process $[53,54]$. In fact, oxidative stress and inflammatory response are considered the main mechanisms involved in the damages caused by the ethanol consumption $[1,52,54-56]$.

Ethanol exposure also leads to oxidative stress by other pathways, such as direct action on biological membranes of cells and organelles, i.e., mitochondria $[52,57]$. Briefly, the lesion in the mitochondria membrane provokes dysfunction of the organelle, causing the overproduction of ROS and reduced production of ATP, which displays apoptosis [52, 57-59]. In addition, ethanol activates NADPH oxidase (NOX), which is highly expressed by glial cells, which provokes high concentrations of ROS in the CNS and neuronal cells death $[1,47,52,60]$. Among the brain regions impaired, motor cortex, nuclei of the base, and cerebellum, that play a pivotal role on motor performance, can be highlighted, which reflects the poor motor performance by ethanol-intoxicated animals [4]. Besides the motor function, emotional behavior alterations are in accordance of damage on mesocorticolimbic system (i.e., ventral tegmental area, nucleus accumbens, amygdala, prefrontal cortex, and hippocampus), which leads to emotional disorders, i.e., depression and anxiety, as well as memory deficits [3, 54, 60-63]. In our results, animals treated with AEG1 displayed the behavioral profile related to control subjects on behavioral tasks, revealing that the mushroom has properties that counteract these damages generated by ethanol exposure.

In the findings on the REDOX balance in the ethanoltreated animals, we have evidenced an imbalance of this system, with an increase in ROS generation, culminating in oxidative stress due to lipid peroxidation, which could be observed in the MDA levels, a biomarker of oxidative stress that is indicative of damage to the plasma membrane [64, 65], that have been demonstrated by our group previously $[9,10]$. On the other hand, animals treated with AEG1 obtained the normalization of the MDA levels, in which we hypothesize that the extract was able to reduce the damage on the biological membranes generated by the unbalance in the REDOX system after the consumption of ethanol. Other interesting data found in this study, that corroborate with the idea that AEG1 was able to reduce oxidative stress, are the results of GSH levels and TEAC. Such markers consist of antioxidant defenses.

The GSH, an important marker of cellular oxidative stress, has several antioxidant functions, serving as a cofactor of Glutathione Peroxidase (GPx) in the detoxification of hydrogen peroxide $(\mathrm{H} 2 \mathrm{O} 2)$, that in turn reduces reactive species $[66,67]$. The TEAC, as already mentioned, measures total antioxidant capacity, which will include elements such as the Alpha-tocopherol, and that its main role is to inhibit lipoperoxidation reactions, being assisted by GSH [67]. We suppose that ethanol intoxication in the binge pattern elicits a significant elevation of GSH and TEAC levels due to an exacerbated physiological response in the attempt to compensate for the high production of reactive species and to reduce neuronal damage $[54,68]$. Therefore, high production and consumption of these antioxidant markers can lead to failure of REDOX defense systems, culminating in severe oxidative stress [69]. In contrast, the AEGl was able to normalize these levels of antioxidants of the animals of the treated group, demonstrating that it elicits mechanisms to reduce oxidative damage, supporting that endogenous production of antioxidants defenses were controlled, remained at the same level of the control group.

In fact, some studies demonstrate that G. lucidum reduces oxidative damage and suppresses lipid peroxidation $[56,70]$. Studies claim that this ability of G. lucidum is due to a large number of bioactive components present in the mushroom, such as ergosterol, flavonoids, unsaturated fatty acids, ganodermic acid (triterpene), and polysaccharides [70-74].

Polysaccharides are bioactive compounds that have been reported as an important neuroprotective molecule, acting as antioxidants [75]. The polysaccharides $\beta(1,3)$ and $(1,6)-\mathrm{D}$ glucans are the main components of these mushrooms [76]. In addition, a study demonstrated that animals treated with 
G. lucidum polysaccharide reduced apoptosis related to mitochondria in animal models of renal failure, demonstrating that this bioactive compound restores the mitochondrial function [58]. Besides, G. lucidum polysaccharide inhibited the translocation of $\mathrm{p} 47$ phox, which consists of a neutrophil NADPH oxidase enzyme-forming subunit, responsible for the formation of the superoxide anion to the membrane, reducing the production of enzyme-dependent superoxide [57]. Finally, a study found that pretreatment with G. luci$d u m$ at a dose of $100 \mathrm{mg} / \mathrm{kg}$ promoted an improvement in energy metabolism and balance between inhibitory and excitatory neurotransmitters in the CNS of rats treated with ethanol [77], with reflects on behavioral profile.

Thus, we postulate that G. lucidum is able to reduce lipid peroxidation and consequent damages on the cells through oxidative stress mechanisms. In addition, we believe that occurs a reduction of the ROS generation by mitochondria after the treatment with the mushroom, due to its ability to improve the performance of mitochondrial activity, explaining the reduction of the requirement for compensatory exacerbated endogenous production of antioxidant defenses, returning to the levels of GSH and TEAC to baseline in the animals of the treated group.

In addition to the antioxidant activity, recent studies have shown that G. lucidum has immunomodulatory potential [78]. A study demonstrated that G. lucidum is able to inhibit TNF $\alpha$, IL- $1 \mathrm{~b}$, and IL- 6 from cortical microglial cells, suppressing NF- $\kappa$ B signaling [79]. It is also reported that G. lucidum polysaccharides can modulate the functions of many immune system components, such as antigen-presenting cells, T and B lymphocytes, NK cells, neutrophil granulocytes, dendritic cells, and cytokine production, demonstrating anti-inflammatory activity [72]. In this sense, we also believe that G. lucidum could also act by reducing neuroinflammatory responses.

Thus, we claim that G. lucidum promotes greater viability of the CNS cells, through reduced damage from oxidative stress and neuroinflammation, that consist of two important ethanol-induced deleterious effects mechanisms, which reflects in better performance of treated animals in all behavioral tests performed. In this way, we demonstrated that the mushroom has potential activities against the damages caused by alcohol consumption, characterizing the functional food as an important element that can be used as an alternative for the management of alcoholic disorders. Moreover, G. lucidum is a mushroom that has been used for years as a nutraceutical for the prevention or treatment of diseases of the most varied origin, which has the advantage of being used as a functional food. It is perceived here the timely viability of the use of this natural source as a food supplement to support on the management of alcohol-related disturbances as an adjuvant in therapeutics, since it presented low toxicity and interesting biological effects [74, 80, 81].

\section{Data Availability}

The behavioral and oxidative data used to support the findings of this study are available from the corresponding author upon request.

\section{Conflicts of Interest}

The authors declare that they have no conflicts of interest.

\section{Acknowledgments}

The authors thank Fundação de Apoio à Pesquisa do Pará (FAPESPA). In addition, we thank Programa de Apoio à Pós-Graduação Coordenação de Aperfeiçoamento de Pessoal de Nível Superior (CAPES) by PROCAD financial support. This work was also supported by Pró-Reitoria de Pesquisa e Pós-Graduação da UFPA (PROPESP, UFPA, Brazil) on open access publication fees. CSFM and MCM are supported by a research fellowship from $\mathrm{CNPq}$.

\section{References}

[1] F. T. Crews and K. Nixon, "Mechanisms of Neurodegeneration and Regeneration in Alcoholism," Alcohol and Alcoholism, vol. 44, no. 2, pp. 115-127, 2009.

[2] G. B. Oliveira, E. d. A. Fontes Jr., S. de Carvalho et al., "Minocycline mitigates motor impairments and cortical neuronal loss induced by focal ischemia in rats chronically exposed to ethanol during adolescence," Brain Research, vol. 1561, pp. 23-34, 2014.

[3] M. Pascual, A. M. Blanco, O. Cauli, J. Miñarro, and C. Guerri, "Intermittent ethanol exposure induces inflammatory brain damage and causes long-term behavioural alterations in adolescent rats," The European Journal of Neuroscience, vol. 25, no. 2, pp. 541-550, 2007.

[4] F. B. Teixeira, L. N. d. S. Santana, F. R. Bezerra et al., "Chronic ethanol exposure during adolescence in rats induces motor impairments and cerebral cortex damage associated with oxidative stress," PLoS ONE, vol. 9, no. 6, article e101074, 2014.

[5] T. K. Li, "Quantifying the risk for alcohol-use and alcoholattributable health disorders: present findings and future research needs," Journal of Gastroenterology and Hepatology, vol. 23, no. s1, pp. S2-S8, 2008.

[6] T. D. Saha, F. S. Stinson, and B. F. Grant, "The role of alcohol consumption in future classifications of alcohol use disorders," Drug and Alcohol Dependence, vol. 89, no. 1, pp. 82-92, 2007.

[7] V. Berridge, R. Herring, and B. Thom, "Binge Drinking: a Confused Concept and its Contemporary History," Social History of Medicine, vol. 22, no. 3, pp. 597-607, 2009.

[8] National Institute on Alcohol Abuse and Alcoholism NIAAA, Department of Health and Human Services; National Institutes of Health NIAAA Newsletter, National Institute on Alcohol Abuse and Alcoholism. Featuring information from the National Institute on Alcohol Abuse and Alcoholism, Bethesda, MD, EUA, 2004.

[9] I. J. A. Belém-Filho, P. C. Ribera, A. L. Nascimento et al., "Low doses of methylmercury intoxication solely or associated to ethanol binge drinking induce psychiatric-like disorders in adolescent female rats," Environmental Toxicology and Pharmacology, vol. 60, pp. 184-194, 2018.

[10] L. M. P. Fernandes, S. C. Cartágenes, M. A. Barros et al., "Repeated cycles of binge-like ethanol exposure induce immediate and delayed neurobehavioral changes and hippocampal dysfunction in adolescent female rats," Behavioural Brain Research, vol. 350, pp. 99-108, 2018. 
[11] L. G. S. Souza, M. C. S. Menandro, and P. R. M. Menandro, “O alcoolismo, suas causas e tratamento nas representações sociais de profissionais de Saúde da Família," Physis: Revista de Saúde Coletiva, vol. 25, no. 4, pp. 1335-1360, 2015.

[12] L. Letenneur, C. Proust-Lima, A. Le Gouge, J. Dartigues, and P. Barberger-Gateau, "Flavonoid intake and cognitive decline over a 10-year period," American Journal of Epidemiology, vol. 165, no. 12, pp. 1364-1371, 2007.

[13] B. S. Weeks, "Formulations of dietary supplements and herbal extracts for relaxation and anxiolytic action: Relarian $^{\mathrm{TM}}$," Medical Science Monitor, vol. 15, no. 11, pp. RA256RA262, 2009.

[14] L. M. Willis, B. Shukitt-Hale, and J. A. Joseph, "Recent advances in berry supplementation and age-related cognitive decline," Current Opinion in Clinical Nutrition and Metabolic Care, vol. 12, no. 1, pp. 91-94, 2009.

[15] K. Van Dyk and M. Sano, "The Impact of Nutrition on Cognition in the Elderly," Neurochemical Research, vol. 32, no. 4-5, pp. 893-904, 2007.

[16] R. R. M. Paterson, "Ganoderma - A therapeutic fungal biofactory," Phytochemistry, vol. 67, no. 18, pp. 1985-2001, 2006.

[17] R. G. Wasson, Soma: divine mushroom of immortality, (Harcourt, brace, Jovanovich, Inc, 1698.

[18] S. C. Lin, Medicinal Fungi of China-Production and Products Development, Chinese Agricultural Press, Beijing, 2000.

[19] H. Matsuzaki, Y. Shimizu, N. Iwata et al., "Antidepressant-like effects of a water-soluble extract from the culture medium of Ganoderma lucidum mycelia in rats," BMC Complementary and Alternative Medicine, vol. 13, no. 1, 2013.

[20] S. Wachtel-galor, Y.-t. Szeto, B. Tomlinson, and I. F. Benzie, "Ganoderma lucidum('Lingzhi'); acute and short-term biomarker response to supplementation," International Journal of Food Sciences and Nutrition, vol. 55, no. 1, pp. 75-83, 2009.

[21] B.-J. You, N. Tien, M.-H. Lee et al., "Induction of apoptosis and ganoderic acid biosynthesis by cAMP signaling in_Ganoderma lucidum_," Scientific Reports, vol. 7, no. 1, p. 318, 2017.

[22] S. Choi, V. T. Nguyen, N. Tae et al., "Anti-inflammatory and heme oxygenase-1 inducing activities of lanostane triterpenes isolated from mushroom _Ganoderma lucidum_ in RAW264.7 cells," Toxicology and Applied Pharmacology, vol. 280, no. 3, pp. 434-442, 2014.

[23] Q. P. Chu, L. E. Wang, X. Y. Cui et al., "Extract of Ganoderma lucidum potentiates pentobarbital-induced sleep via a GABAergic mechanism," Pharmacology, Biochemistry, and Behavior, vol. 86, no. 4, pp. 693-698, 2007.

[24] X. Y. Cui, S. Y. Cui, J. Zhang et al., "Extract of Ganoderma lucidum prolongs sleep time in rats," Journal of Ethnopharmacology, vol. 139, no. 3, pp. 796-800, 2012.

[25] K. Socala, D. Nieoczym, K. Grzywnowicz, D. Stefaniuk, and P. Wlaz, "Evaluation of anticonvulsant, antidepressant-, and anxiolytic-like effects of an aqueous extract from cultured mycelia of the Lingzhi or Reishi medicinal mushroom Ganoderma lucidum (higher Basidiomycetes) in mice," Int. J. Med. Mushrooms., vol. 17, no. 3, pp. 209-218, 2015.

[26] M. H. Malone, "The pharmacological evaluation of natural products - general and specific approaches to screening ethnopharmaceuticals," Journal of Ethnopharmacology, vol. 8, no. 2, pp. 127-147, 1983.

[27] R. D. Porsolt, M. Le Pichon, and M. Jalfre, "Depression: a new animal model sensitive to antidepressant treatments," Nature, vol. 266, no. 5604, pp. 730-732, 1977.
[28] S. Percário, A. C. C. Vital, and F. Jablonka, "Dosagem do Malondialdeído," Newslab, vol. 2, pp. 46-50, 1994.

[29] R. Re, N. Pellegrini, A. Proteggente, A. Pannala, M. Yang, and C. Rice-Evans, "Antioxidant activity applying an improved ABTS radical cation decolorization assay," Free Radical Biology \& Medicine, vol. 26, no. 9-10, pp. 1231-1237, 1999.

[30] N. J. Miller, C. Rice-Evans, M. J. Davies, V. Gopinathan, and A. Milner, "A novel method for measuring antioxidant capacity and its application to monitoring the antioxidant status in premature neonates," Clinical Science, vol. 84, no. 4, pp. 407412, 1993.

[31] J. M. McCord and I. Fridovich, "Superoxide dismutase. An enzymic function for erythrocuprein (hemocuprein)," The Journal of Biological Chemistry, vol. 244, no. 22, pp. 60496055, 1969.

[32] T. R. Schalcher, R. S. Borges, M. D. Coleman et al., "Clinical oxidative stress during leprosy multidrug therapy: impact of dapsone oxidation," PLoS ONE, vol. 9, no. 1, p. e85712, 2014.

[33] J. C. Defries, J. P. Hegmann, and M. W. Weir, "Open-field Behavior in Mice: Evidence for a Major Gene Effect Mediated by the Visual System," Science, vol. 154, no. 3756, pp. 15771579, 1966.

[34] T. Karl, R. Pabst, and S. Von-Hörsten, "Behavioral phenotyping of mice in pharmacological and toxicological research," Experimental and Toxicologic Pathology, vol. 55, no. 1, pp. 69-83, 2003.

[35] J. F. Cryan, A. Markou, and I. Lucki, "Assessing antidepressant activity in rodents: recent developments and future needs," Trends in Pharmacological Sciences, vol. 23, no. 5, pp. 238245, 2002.

[36] I. Izquierdo, L. A. Izquierdo, D. M. Barros et al., "Differential involvement of cortical receptor mechanisms in working, short-term and long-term memory," Behavioural Pharmacology, vol. 9, no. 5, pp. 421-427, 1998.

[37] C. D. S. F. Maia, G. M. R. de Souza Lucena, P. B. F. Corrêa et al., "Interference of ethanol and methylmercury in the developing central nervous system," Neurotoxicology, vol. 30, no. 1, pp. 23-30, 2009.

[38] Dossiê antioxidantes, "Food Ingredients Brasil, No. 6," 2009, http://www.unirio.br/ib/dmp/nutricao-integral/arquivos/ fontes-de-consulta-complementar/Antioxidantes $\% 20-\%$ 20FOOD\%20INGREDIENTS\%20BRASIL\%20No6\%20-\% 202009.pdf.

[39] OECD- Organisation for Economic Cooperation and Development, Guidelines for the Testing of Chemicals, OECD 423. Acute Oral Toxicity-Acute Toxic Class Method, Organisation for Economic Cooperation and Development, Paris, 2001.

[40] OECD - Organisation for Economic Cooperation and Development, "Harmonized integrated hazard classification system for human health and environmental effects of chemical substances as endorsed by the 28th joint meeting of the chemicals committee and the working party on chemicals in November," Part, vol. 2, p. 11, 1998.

[41] K. S. Bishop, C. H. J. Kao, Y. Xu, M. P. Glucina, R. R. M. Paterson, and L. R. Ferguson, "From 2000 years of _Ganoderma lucidum_ to recent developments in nutraceuticals," Phytochemistry, vol. 114, pp. 56-65, 2015.

[42] L. C. Cunha, F. S. Azeredo, A. C. V. Mendonça et al., "Avaliação da toxicidade aguda e subaguda, em ratos, do extrato etanólico das folhas e do látex de Synadenium umbellatum Pax," Revista Brasileira de Farmacognosia, vol. 19, no. 2A, 2009. 
[43] A. P. Santos, M. B. Bandeira, and L. O. Siqueira, "Comparação entre diversos métodos de contagem diferencial de leucócitos em pacientes leucopênicos," Revista Brasileira de Hematologia e Hemoterapia, vol. 31, no. 3, pp. 203-205, 2009.

[44] T. Oduola, A. Ngaski, H. Mahe, A. Muhammed, M. Akiibinu, and A. Ajayi, "Thyrotoxic evaluation and lipid peroxidation in wistar albino rats exposed to vitellaria paradoxa stem bark," British Journal of Pharmaceutical Research, vol. 10, no. 4, pp. 1-7, 2016.

[45] K. Abernathy, L. J. Chandler, and J. J. Woodward, "Alcohol and the prefrontal cortex," International Review of Neurobiology, vol. 91, pp. 289-320, 2010.

[46] S. K. Blaine, V. Milivojevic, H. Fox, and R. Sinha, "Alcohol effects on stress Pathways," Canadian Journal of Psychiatry, vol. 61, no. 3, pp. 145-153, 2016.

[47] C. Contet, A. Kim, D. le et al., " $\mu$-Opioid receptors mediate the effects of chronic ethanol binge drinking on the hippocampal neurogenic niche," Addiction Biology, vol. 19, no. 5, pp. 770780, 2014.

[48] A. Belmer, O. L. Patkar, V. Lanoue, and S. E. Bartlett, "5-HT1A receptor-dependent modulation of emotional and neurogenic deficits elicited by prolonged consumption of alcohol," Scientific Reports, vol. 8, no. 1, p. 2099, 2018.

[49] F. T. Crews and R. P. Vetreno, "Neuroimmune Basis of Alcoholic Brain Damage," International Review of Neurobiology, vol. 118, pp. 315-357, 2014.

[50] N. W. Gilpin, K. Misra, M. A. Herman, M. T. Cruz, G. F. Koob, and M. Roberto, "Neuropeptide Y opposes alcohol effects on aamma-aminobutyric acid release in amygdala and blocks the transition to alcohol dependence," Biological Psychiatry, vol. 69, no. 11, pp. 1091-1099, 2011.

[51] X. Liu, K. P. Connaghan, Y. Wei, Z. Yang, M. D. Li, and S. L. Chang, "Involvement of the hippocampus in binge ethanolinduced spleen atrophy in adolescent rats," Alcoholism, Clinical and Experimental Research, vol. 40, no. 7, pp. 1489-1500, 2016.

[52] M. A. Collins and E. J. Neafsey, "Ethanol and adult CNS neurodamage: oxidative stress, but possibly not excitotoxicity," Frontiers in Bioscience, vol. E4, no. 4, pp. 1358-1367, 2012.

[53] X.-x. Dong, Y. Wang, and Z.-h. Qin, "Molecular mechanisms of excitotoxicity and their relevance to pathogenesis of neurodegenerative diseases," Acta Pharmacologica Sinica, vol. 30, no. 4, pp. 379-387, 2009.

[54] C. Tapia-Rojas, F. J. Carvajal, R. G. Mira et al., “Adolescent binge alcohol exposure affects the brain function through mitochondrial impairment," Molecular Neurobiology, vol. 55, no. 5, pp. 4473-4491, 2018.

[55] A. M. White, D. B. Matthews, and P. J. Best, "Ethanol, memory, and hippocampal function: a review of recent findings," Hippocampus, vol. 10, no. 1, pp. 88-93, 2000.

[56] K.-L. Wong, H.-H. Chao, P. Chan, L.-P. Chang, and C.-F. Liu, "Antioxidant activity of Ganoderma lucidum in acute ethanolinduced heart toxicity," Phytotherapy Research, vol. 18, no. 12, pp. 1024-1026, 2004.

[57] D. Zhong, H. Wang, M. Liu et al., “_Ganoderma lucidum_ polysaccharide peptide prevents renal ischemia reperfusion injury via counteracting oxidative stress," Scientific Reports, vol. 5, no. 1, 2015.

[58] T. Ishrat, M. B. Khan, M. N. Hoda et al., "Coenzyme Q10 modulates cognitive impairment against intracerebroventric- ular injection of streptozotocin in rats," Behav.Brain Res., vol. 171, no. 1, pp. 9-16, 2006.

[59] A. J. Kowaltowski and A. E. Vercesi, "Mitochondrial damage induced by conditions of oxidative stress," Free Radical Biology \& Medicine, vol. 26, no. 3-4, pp. 463-471, 1999.

[60] I. Matsumoto, "Proteomics approach in the study of the pathophysiology of alcohol-related brain damage," Alcohol and Alcoholism, vol. 44, no. 2, pp. 171-176, 2009.

[61] J. Bouayed, H. Rammal, and R. Soulimani, "Oxidative stress and anxiety: relationship and cellular pathways," Oxidative Medicine and Cellular Longevity, vol. 2, no. 2, pp. 63-67, 2009.

[62] T. B. Garcia, K. R. M. Oliveira, J. L. M. do Nascimento et al., "Glutamate induces glutathione efflux mediated by glutamate/aspartate transporter in retinal cell cultures," Neurochemical Research, vol. 36, no. 3, pp. 412-418, 2010.

[63] J. R. Stevenson, J. P. Schroeder, K. Nixon, J. Besheer, F. T. Crews, and C. W. Hodge, "Abstinence following alcohol drinking produces depression-like behavior and reduced hippocampal neurogenesis in mice," Neuropsychopharmacology, vol. 34, no. 5, pp. 1209-1222, 2009.

[64] W. Liu, Z. Xu, Y. Deng et al., "Excitotoxicity and oxidative damages induced by methylmercury in rat cerebral cortex and the protective effects of tea polyphenols," Environmental Toxicology, vol. 29, no. 3, pp. 269-283, 2014.

[65] T. Yang, Z. F. Xu, W. Liu et al., "Alpha-lipoic acid protects against methylmercury-induced neurotoxic effects via inhibition of oxidative stress in rat cerebral cortex," Environmental Toxicology and Pharmacology, vol. 39, no. 1, pp. 157-166, 2015.

[66] S. J. Cha, H. Kim, H.-J. Choi, S. Lee, and K. Kim, "Protein Glutathionylation in the Pathogenesis of Neurodegenerative Diseases," Oxidative Medicine and Cellular Longevity, vol. 2017, 9 pages, 2017.

[67] A. A. Jordão-Júnior, P. G. Chiarello, M. S. M. Bernardes, and H. Vannucchi, "Lipid Peroxidation and Ethanol: Role of Reduced Glutation and Vitamin and," Medicina (Ribeirao Preto. Online), vol. 31, no. 3, pp. 434-449, 1998.

[68] M. F. Reis and C. L. M. S. C. Rocha, "Medicinal mushrooms: review on bioactive compounds and biological effects," SaBiosRevista de Saúde e Biologia, vol. 10, no. 1, pp. 49-164, 2015.

[69] M. Maes, N. De Vos, R. Pioli et al., "Lower serum vitamin E concentrations in major depression: Another marker of lowered antioxidant defenses in that illness," Journal of Affective Disorders, vol. 58, no. 3, pp. 241-246, 2000.

[70] M. Okazaki, N. Iwata, S. Horiuchi et al., "Protective effects of a water-soluble extract from culture medium of Ganoderma lucidum mycelia against neuronal damage after hypoxia-ischemia in mice," Japanese Journal of Complementary and Alternative Medicine, vol. 5, no. 2, pp. 153-162, 2008.

[71] M. Fukuzawa, R. Yamaguchi, I. Hide et al., "Possible involvement of long chain fatty acids in the spores of Ganoderma lucidum (Reishi Houshi) to its anti-tumor activity," Biological \& Pharmaceutical Bulletin, vol. 31, no. 10, pp. 1933-1937, 2008.

[72] J. Habijanic, M. Berovic, B. Boh, M. Plankl, and B. Wraber, "Submerged cultivation of _Ganoderma lucidum_ and the effects of its polysaccharides on the production of human cytokines TNF- $\alpha$, IL-12, IFN- $\gamma$, IL-2, IL-4, IL-10 and IL-17," New Biotechnology, vol. 32, no. 1, pp. 85-95, 2015. 
[73] T. Wu and B. Xu, "Antidiabetic and antioxidant activities of eight medicinal mushroom species from China," Int. J. Med. Mushrooms., vol. 17, no. 2, pp. 129-140, 2015.

[74] J. P. Yuan, J. H. Wang, and X. Liu, "Distribution of free and esterified ergosterols in the medicinal fungus Ganoderma lucidum," Applied Microbiology and Biotechnology, vol. 77, no. 1, pp. 159-165, 2007.

[75] K. Li, K. Na, T. Sang, K. Wu, Y. Wang, and X. Wang, "The ethanol extracts of sporoderm-broken spores of Ganoderma lucidum inhibit colorectal cancer in vitro and in vivo," Oncology Reports, vol. 38, no. 5, pp. 2803-2813, 2017.

[76] S.-H. Wang, C.-J. Liang, Y.-W. Weng et al., "Ganoderma lucidum polysaccharides prevent platelet-derived growth factor-stimulated smooth muscle cell proliferation in vitro and neointimal hyperplasia in the endothelial-denuded artery in vivo," Journal of Cellular Physiology, vol. 227, no. 8, pp. 3063-3071, 2012.

[77] O. B. Shevelev, A. E. Akulov, A. S. Dotsenko et al., "Neurometabolic effect of Altaian FungusGanoderma lucidum(Reishi mushroom) in rats under moderate alcohol consumption," Alcoholism, Clinical and Experimental Research, vol. 39, no. 7, pp. 1128-1136, 2015.

[78] C. Ma, S.-H. Guan, M. Yang, X. Liu, and D.-A. Guo, “Differential protein expression in mouse splenic mononuclear cells treated with polysaccharides from spores of Ganoderma lucidum," Phytomedicine, vol. 15, no. 4, pp. 268-276, 2008.

[79] B. Chi, S. Wang, S. Bi et al., "Effects of ganoderic acid A on lipopolysaccharide-induced proinflammatory cytokine release from primary mouse microglia cultures," Experimental and Therapeutic Medicine, vol. 15, no. 1, pp. 847-853, 2017.

[80] E. Ernst, "American herbal pharmacopoeia: botanical Pharmacognosy - microscopic characterization of botanical medicines," Focus on Alternative and Complementary Therapies., vol. 17, no. 1, 2012.

[81] S. P. Wasser, "Reishi or ling zhi (Ganoderma lucidum)," Encyclopedia of dietary supplements, vol. 1, pp. 603-622, 2005. 